\title{
Neurobiological studies within the framework of highly technological teaching
}

\author{
Marianna Ababkova ${ }^{1 *}$, and Veronica Leontyeva ${ }^{1}$ \\ ${ }^{1}$ Peter the Great St. Petersburg Polytechnic University, 195251 Polytechnicheskaya st. 29, Russian \\ Federation
}

\begin{abstract}
Modern highly technological teaching widely uses neurotechnologies related to creating new educational products, expanding the content of education and establishing objective feedback with students. One of the most promising neurotechnologies in the sphere of education is the method of biological feedback (BFB) based on studying the current psychophysiological state of students and using this information for further correction of the educational process, improving its quality and efficiency. This article presents the results of the studies based on the method of biological feedback to investigate the influence of the form of presentation of the educational material on the current psychophysiological state of students. During the BFB-studies, we used the PEN method developed by Hans and Sybil Eysenck to form focus groups of students and the CMS method to process the results of the studies. The research resulted in quantitative values of parameters of the current psychophysiological state of students reflecting the degree of intensity of such properties as the general adaptive resource, the degree of mobility (instability) of psychological processes, neuroticity, psychic productivity etc. (a total of 19 parameters).The study's results based on the biological feedback method showed that studying educational materials that are different in form influences the parameters of the current psychophysiological state of students. The indicators of reserve of control of psychological activity, regulating and adapting the psychological sphere, as well as the indicators of reserve of syndromologicaladaptiveness of the current state if students study the textual material on their own, will improve as compared to studying graphical material only. Different types of educational material can cause anxiety and increased apprehension, with textual material contributing the least to these effects.
\end{abstract}

\section{Introduction}

Development of highly technological teaching is related to such key cognitive technologies of the future as neuro-interfaces, Neuronet and biological feedback (BFB). Using devices based on neuro-technology will make it possible to create new educational products, expand the content of education by transiting from the textual, graphical or audio content of the educational process to include tactile, motoric, emotional and other content. Developing

*Corresponding author: ababkova_myu@spbstu.ru 
and copying physiologic interfaces that can be used to improve the activities in the spheres of corporative marketing, medicine, sports, gaming etc., the potential of the entire body (body position and facial expression scanners - Kinect, LeapMotion, MYO etc.), sight direction (Eye Tribe), controllers of the current health state (heartbeat frequency, blood pressure), monitors of the level of physical activeness (Fitbit, Jawbone), electric encephalographs for sending messages (Emotiv) will inevitably lead this set of tools to become widely used in the sphere of education in the near future [1].

Neurotechnologies using biological feedback are more and more widely used in the modern educational process [2]. The market of evaluation and training cognitive abilities based on BFB methods, which was USD 1.7 billion in 2013, is expected to grow to USD 5.7 billion by 2018 , according to predictions of experts [3].

The essence of the BFB method is visualization for the tested person using a screen, or in the audio form, of the current values of his/her physiological parameters determined by the clinical protocol, that are divided into two large groups:

- "neuronic feedback" (neurofeedback, neurotherapy) based on modifying different parameters of the electroencephalogram (EEG) of the brain (amplitude, power, coherence etc. of the basic rhythms of EEG;

-biological feedback (biofeedback) measuring the values of the vegetative (sympathicparasympathetic) activation (skin conductivity, cardiogram, pulse frequency, electromyogram, temperature, photopletizmogram etc.).

\section{Overview of literature}

Currently, BFB methods are used in education in the following cases:

- achieving high functional ability of participants of the educational process (studying adaptability of students to the educational process [4], measuring the actual psychologicalstate of high school pupils in the conditions of interactive studying [5], relieving apprehension of students during the exams, relaxation and positive attitude, speech correction) [6];

- optimizing physical or mental activity (general improving of health and harmonizing the performance of the nervous and vascular systems);

- therapy of addictive behavior, treatment of hyper-active schoolchildren [7].

In the foreign literature there are articles describing the use of BFB methods in education $[8,9,10]$.

In some foreign educational organizations, teachers and students are offered courses of BFB for health improving and harmonizing the nervous and vascular systems. For example, in Japanese universities, BFB methods are used during exam periods to relieve students' apprehension. In schools of the state of South Dakota (USA), these methods are used within the framework of the program "Self-Awareness through Training with BFB Methods" [7]. The Charity fund Melinda \& Bill Gates Foundation (specializing in improving the educational system) issued a grant in 2011 for Clemson University (South Carolina, USA) to carry out pilot studies of students' involvement into the educational process by changing the galvanic resistance of skin [11].

Russian specialists, like their foreign colleagues, actively investigate projects based on BFB methods. For example, a monitoring research on students' adaptedness for studying at Saint-Petersburg polytechnic university Named after Peter the Great showed that the process of students' adaptation for studying at a technical higher school can be deteriorated not only due to their particular features in the emotional and communicative spheres but also due to various deficiencies in the development of their intellectual capabilities, the most important of which is the logical, abstract and 3D engineering thinking [4]. 
Within the framework of another project, the actual psychological state of senior high school students was studied in the conditions of interactive teaching. Differences were detected in well-being, activeness and mood in class with interactive methods of introducing new educational material [5].

With the use of BFB-based diagnostics of physiological changes for students in the conditions of educational stress, methods of stress states correction are being developed in relation to teaching [12].

Focused instrumental measurement of the psychophysiological state of students will help find out which time of day and which conditions are the most favorable for studying and tune individual regimes of the educational process, as well as efficiently control the well-being of students and teachers [1].

Therefore, BFB methods can be used as a means to promote an individual method of improving the well-being, as a tool that can help to bring up healthy generations, as a set of methods and forms of education without negative impact on the state of health, as an efficient method to evaluate pedagogical technologies [13].

\section{Method of studies}

Within the framework of the research carried out by the "Advertising and PR" department of the Institute of Humanities of Saint-Petersburg polytechnic university named after Peter the Great in the spring of 2017, a study was conducted to evaluate the emotional state of the 2 grade students of the course "Advertising and PR" in the practical seminars on the "Basics of Advertising". The purpose of the study was investigating the inter-relation between the forms of presenting the educational material and the current psychological state of the students using the measured results with the method of biological feedback. The tasks of the study were as follows:

- preliminary evaluation of the psychophysiological state of students on the basis of BFB method before the educational presentation.

- final evaluation of the psychophysiological state of students on the basis of BFB method after the educational presentation.

- comparing the obtained data for the experimental and the reference groups and forming the conclusions.

A hypothesis for the study was the assumption that presenting the educational material in the graphical form improves comprehension and memorizing, has a positive effect on the psychological state of students as compared with the textual form of presentation.

For the experiment, two focus groups of students were formed, equal in the number (12 people each), similar in the age and sex. Besides, the participants had similar distribution in psychological parameters estimated with the help of a PEN questionnaire to measure such psychological features as neuropsychological instability, extraversion and psychoticism.

Collecting the data on the emotional state of students was based on psychophysiological methods of CMS (Current Mental State - of the current psychological state) implemented in the form of a hardware-software complex (HSC) for CMS. This method makes it possible to carry out an objective evaluation of the psychological state by the data from cardiorhythmography, as well as monitor the changes in the state during various time periods and evaluate the effect of various influences onto the psychological state. The hardware-software complex includes a microcardioanalyzer and CMS software. The results of the investigation were quantitative (in per cent) values for parameters of the current psychological state of the examined persons (in 19 scales) reflecting the degree of the feature of the current psychological state. In these scales (in per cent) the similarity or difference is determined between the state of the examined person and the reference samples. 


\section{Processing the studies' results}

Before class, both focus groups passed a preliminary evaluation of the psychophysiological state with the biological feedback method on the basis of the HSC for CMS. Then each group was given a lesson with studying theoretical material presented in various forms. The experimental group was given a lesson in self-studying of the material on the "Basics in advertising" in the direction "Brand mapping. Compiling Brand Comprehension Maps" in the form of a presentation containing graphical illustrative material (animated schemes, tables), video and sound. The reference focus group was given the same theoretical material but presented in the textual form only. The students studied the theoretical material on their own during 45 minutes, without interference from the tutor. After independently studying the theoretical material, the experimental and the reference groups were given a theoretical test to check their comprehension of the educational material on the topic "Brand mapping. Compiling Brand Comprehension Maps".

It was expected that the experimental group would have better results of the theoretical test after self-study of the presentation. However, the average mark of the theoretical testing of the experimental group was 2.91, with 4 "poor" marks, 5 "satisfactory" marks and 3 "good" marks, with no "excellent" marks. The reference group had the average mark 3.25, with 4 "poor" marks, 3 "satisfactory" marks, 3 "good" marks and 2 "excellent" marks. In our opinion, such results can be related to student poor ability to work with diagrams and tables, as well as lack of comments and explanations from the tutor. It appears that, for efficient studying of theoretical materials containing diagrams, tables and video, a somewhat different approach is required than for studying textual materials.

After studying theoretical materials, each focus group was investigated again with the psychophysiological method of CMS by the data from cardiorhythmography. Then the data was entered into a computer and processed with the specialized software (Fig. 1).

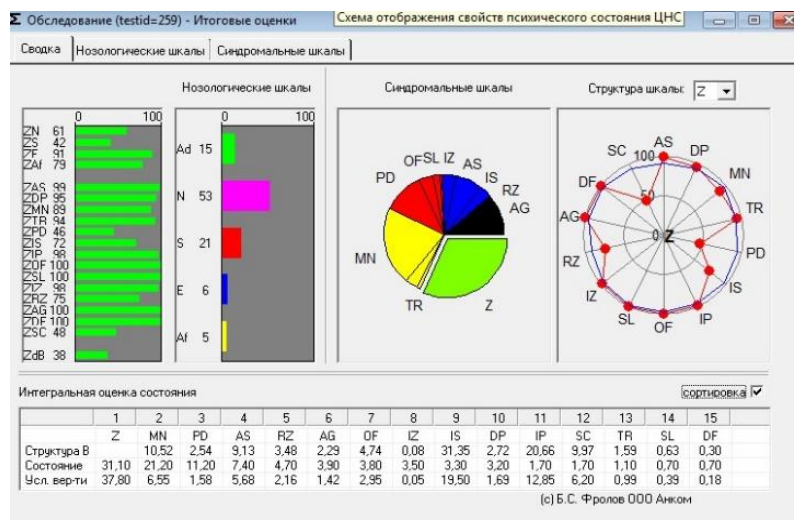

Fig. 1.A screenshot of the results of testing with the CMS software.

Table 1 shows the main average marks for the experimental and reference groups by the results of data processing comparable to reference values. 
Table 1.Summary of the experiment results.

\begin{tabular}{|l|c|c|c|c|c|c|c|c|c|c|}
\hline \multirow{2}{*}{ Experiment number } & \multicolumn{4}{|c|}{ Nosological scales } & \multicolumn{5}{c|}{ Syndromological scales } \\
\cline { 2 - 12 } & Af & E & S & Ad & N & IS & MN & PD & OF & Z \\
\hline 1.1 & 14.83 & 13.55 & 16.11 & 15.22 & 7.16 & 5.38 & 12.44 & 7.86 & 5.7 & 15.10 \\
\hline 1.2 & 16.94 & 7.72 & 10.66 & 23.33 & 7.94 & 2.58 & 5.52 & 5.41 & 3.74 & 28.63 \\
\hline 2.1 & 20.69 & 21.53 & 28.38 & 21 & 8.30 & 6.62 & 22.67 & 10.41 & 4.41 & 20.81 \\
\hline 2.2 & 23.61 & 19.15 & 9.46 & 35.77 & 11.69 & 3.34 & 14.45 & 5.93 & 4.84 & 35.84 \\
\hline Reference value & 4.8 & 7.6 & 6.3 & 73.3 & 8.1 & 1 & 1.1 & 1.3 & 1.1 & 88.4 \\
\hline
\end{tabular}

The overall compensation of the state of students groups. This value reflects the reserve of control of the psychological activity, the optimal adaptive regulation (and adaptation) of the mind. Before the experiment, the experimental group showed a larger limitation of the

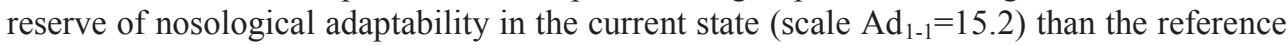
group (scale $\mathrm{Ad}_{2-1}=21$ ), with the reference norm $\mathrm{Ad}=73.3$ (Fig. 2).

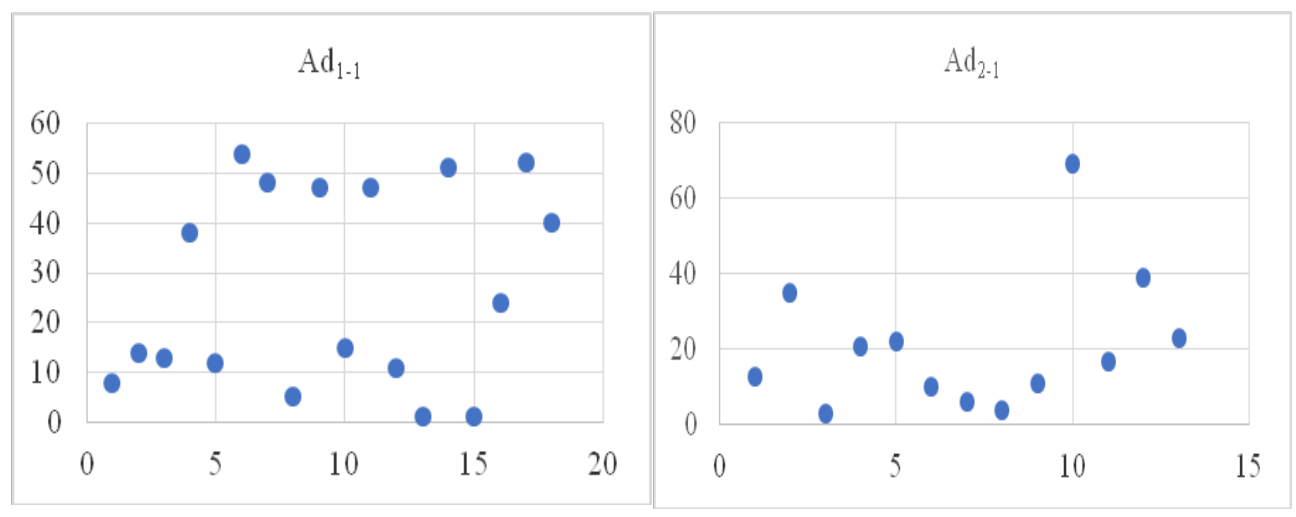

Fig. 2. Comparison of the values for the reserve of adaptability Ad for the experimental and reference groups before studying the educational materials.

We can also note the current limitation of the reserve of syndromological adaptability in the current state for the experimental group $Z_{1-1}=15.10$ (this value for the reference group was even higher, $\left.Z_{2-1}=20.81\right)$ as compared to the reference norm $(Z=88.4)$, Fig. 3 .

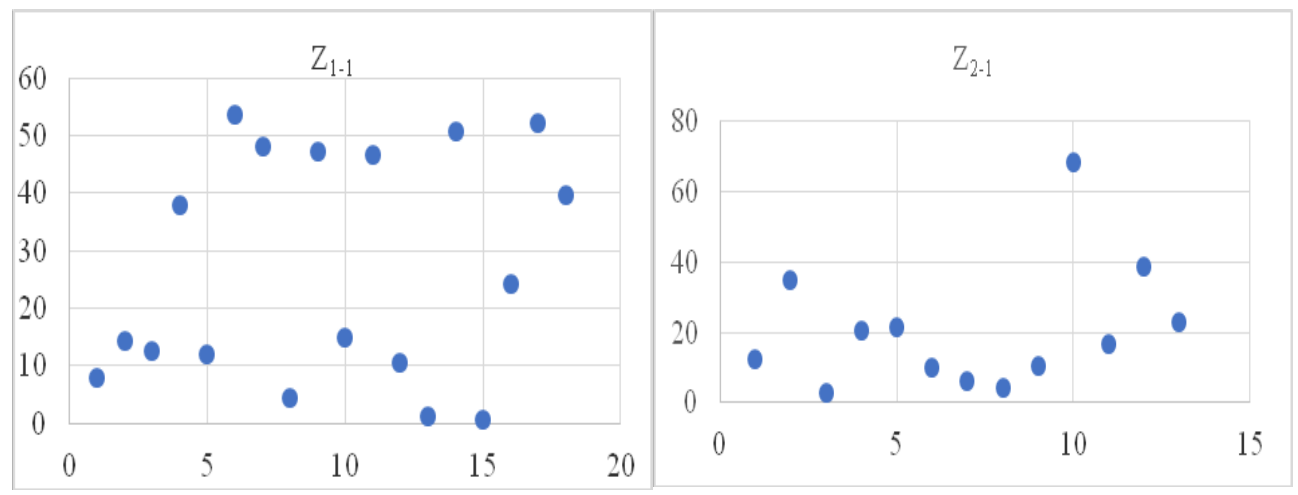

Fig. 3.Comparison of the values for the reserve of syndromological adaptability in the current state $Z$ for the experimental and reference groups before studying the educational materials. 
After studying the educational materials, the values for the reserve of nosological adaptability in the current state for the experimental group slightly increased (scale $\operatorname{Ad}_{1-2}=23.3$ ); for the reference group, the value increased more abruptly ( scale $\operatorname{Ad}_{2-2}=35.77$ ) (Fig. 4).

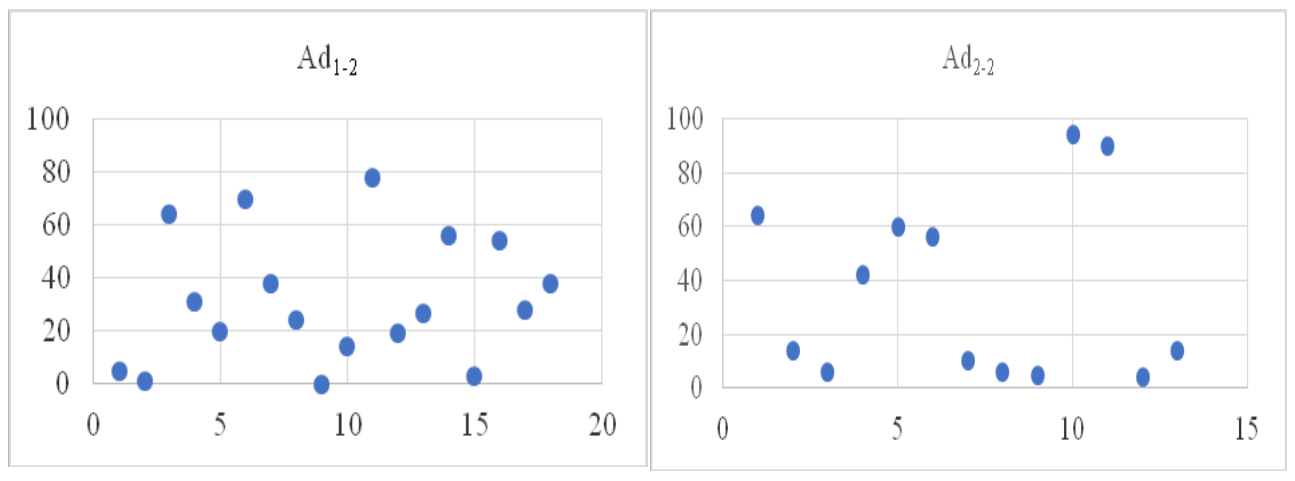

Fig. 4. Comparison of the values for the reserve of adaptability Ad for the experimental and reference groups after studying the educational materials.

The current limitation of the reserve of syndromological adaptability for the experimental group increased $Z_{1-2}=28.63$ (for the reference group, this value increased even more, $Z_{2-2}=35.84$ ), Fig. 5.

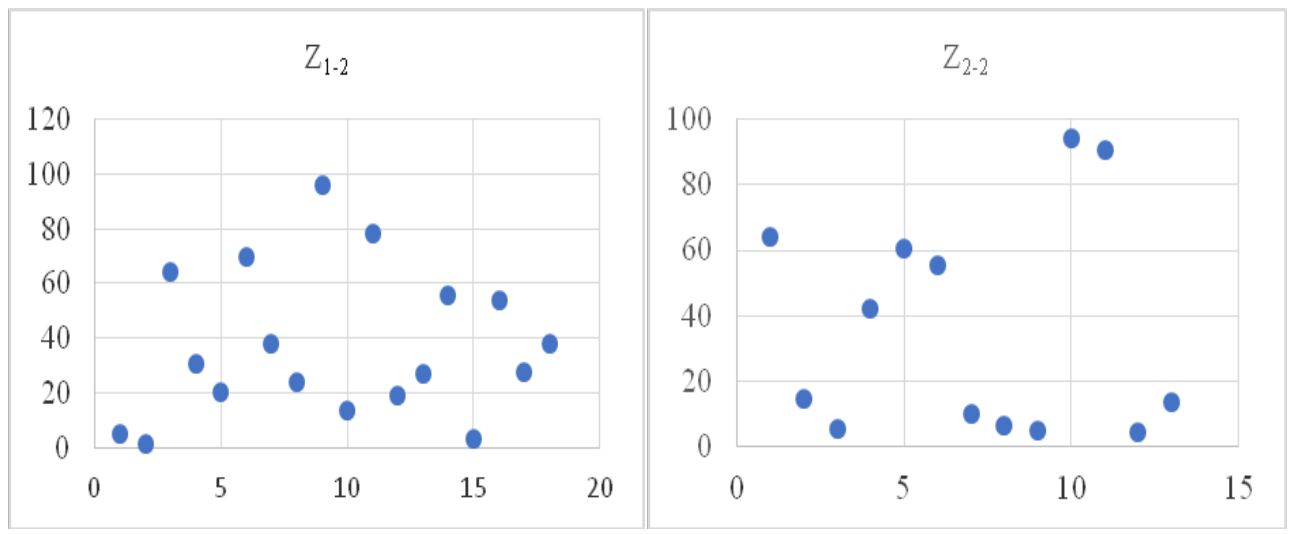

Fig. 5. Comparison of the values for the reserve of syndromological adaptability in the current state $\mathrm{Z}$ for the experimental and reference groups after studying the educational materials.

State of health (psychological and physical). Before the experiment, the experimental group had the average value in the scale of hypochondria equal to 2.01 , and the reference group had the value of 6.5.Before the experiment, both groups had not a very good state of health and state of physical and psychological comfort (the reference value is 1 ), which can be explained by the end of the academic year and general tiredness. However, upon completion of the experiment, this value for the reference group slightly increased (scale IP, the reference group had 5.1, the experimental group had 3.4). Studying new material presented in the schematic form decreased the well-being of the experimental group.

Emotional sphere. This value reflects the character of the emotional reaction or emotional component (mental balance, emotions intensity). Before the class study, for both groups, the value of the emotional (and, therefore, the indirectly-volitional, mental etc.) psychological deviation or stress (scale Af, the reference value of 4.8) was increased 
$\left(\mathrm{Af}_{1-1}=14.8, \mathrm{Af}_{2-1}=20.69\right)$. After the class study, both groups show an increased affectivity value (scale Af, values 16.9 and 23.6, respectively).

In regard of the value DP (depression of the psychological state, opposite to vigor, the feature of depression, the reference value is 1.4), it can be noted that, before the experiment, the experimental group had 2.57, and the reference group had 2.13. After studying the educational material, both groups had slightly decreased values for vigor in the form of inclination for seriousness and apprehension with the generally high level of the positive attitude (scale DP, values $\mathrm{DP}_{1-2}=2.68$ and $\mathrm{DP}_{2-2}=3.13$, respectively).

Before the class, both groups showed a clearly expressed degree of the current excitement, vivacity and intensive emotions, increased movement activity, faster speech and increased self-esteem (scale $\mathrm{MN}_{1-1}=12.4, \mathrm{MN}_{2-1}=22.67$ ) as compared to the reference sample $(\mathrm{MN}=1.1)$. As a result of different forms of the study material, both groups had this value improved and decreased almost twofold $\left(\mathrm{MN}_{1-2}=5.52\right.$ and $\left.\mathrm{MN}_{2-2}=14.45\right)$.

Before the experiment, both groups showed the current limitation of the assuredness, exaggerated trend to be cautious, manifestations of anxiety and apprehension $\left(\mathrm{TR}_{1-1}=1.51\right.$ and $\mathrm{TR}_{2-1}=3.45$, the reference norm being 1.3). Upon completion of the experiment, the experimental group had this value increased, whereas the reference group had it comparable with the reference value $\left(\mathrm{TR}_{1-2}=2.21\right.$ and $\mathrm{TR}_{2-2}=1.3$, respectively).

Before the experiment, the experimental and reference groups showed the current emotional intemperance, disturbed balance "Myself-Others" $\left(\mathrm{IS}_{1-1}=5.38\right.$ and $\mathrm{IS}_{2-1}=6.62$, the reference norm being 1). After the experiment, both groups had this value improved almost twofold $\left(\mathrm{IS}_{1-2}=2.58\right.$ and $\left.\mathrm{IS}_{2-2}=3.34\right)$.

Before the beginning of the class studies, the value of the degree of limitation of the current spontaneity and productivity of the mind when reflecting the reality for the experimental and reference groups differed by several times $\left(\mathrm{SL}_{1-1}=1.63\right.$ and $\mathrm{SL}_{2-1}=4.31$, the reference norm being 1). After the experiment, this value decreased for both groups; for the reference group is came close to the norm $\left(\mathrm{SL}_{1-2}=0.37\right.$ and $\mathrm{SL}_{2-2}=1.31$ ).

Perceptive-thinking sphere. This value reflects the particular features of comprehension and thinking. During the preliminary series of testing, both groups had this value close to the reference value $\left(\mathrm{N}_{1-1}=7.16, \mathrm{~N}_{2-1}=8.3\right.$, the reference norm being 8.1). After the experiment, this value, which characterizes the presence of risk of actual psychotraumatizing emotions, as well as a slight limitation of vulnerability and increased sensitivity remained almost unchanged for the experimental group, whereas for the reference group it was increased as compared to the reference sample $\left(\mathrm{N}_{1-2}=7.94, \mathrm{~N}_{2-2}=\right.$ 11.69).

A well-expressed degree of a decrease in specificity (pragmatism, reality) of thinking before the experiment for the experimental group was lower than that of the reference group; this value for both groups was higher than normal $\left(\mathrm{S}_{1-1}=16.11, \mathrm{~S}_{2-1}=28.38\right.$, the reference norm being 6.3). After classes, this value was improved for both groups; for the reference group it was decreased more and came closer to the normal $\left(\mathrm{S}_{1-2}=10.66\right.$, $\left.\mathrm{S}_{2-2}=9.46\right)$.

Before the experiment, a higher degree of a decrease in self-criticism for own opinion and actions was manifested in both groups $\left(\mathrm{PD}_{1-1}=7.86, \mathrm{PD}_{2-1}=10.41\right.$, the reference norm being 1.3). After classes, the value of assuredness of correctness (reality, accuracy, faultlessness) of own opinion was decreased in both groups, with a more noticeable change in the reference group $\left(\mathrm{PD}_{1-2}=5.41, \mathrm{PD}_{2-2}=5.93\right)$.

Before the experiment, the degree of manifestation of repeated (obsessive, arising unwillingly) emotions, decrease in the ability to willingly change over to other emotions was higher in both groups $\left(\mathrm{OF}_{1-1}=5.7, \mathrm{OF}_{2-1}=4.41\right.$, the reference norm being 1.1); after the experiment, it was decreased for the experimental group, and it slightly increased for the reference group $\left(\mathrm{OF}_{1-2}=3.74, \mathrm{OF}_{2-2}=4.84\right)$. 
Before the experiment, both groups showed some degree of limitation of the current spontaneity, differentiation and productivity of mind when reflecting the reality $\left(\mathrm{SL}_{1-1}=1.63, \mathrm{SL}_{2-1}=4.31\right.$, the reference norm being 1$)$; after the experiment, this value was closer to normal for the reference group $\left(\mathrm{SL}_{1-2}=0.37, \mathrm{SL}_{2-2}=1.35\right)$.

The sphere of will.This value mainly reflects an intention, direction, strength and intensity of psychological reaction. Before the experiment, both groups showed a small degree of slowing down in the psychological reactions; however, the experimental group slowed down more $\left(\mathrm{E}_{1-1}=13.55, \mathrm{E}_{2-1}=21.53\right.$, the reference norm being 7.6). After the experiment, the experimental group had this value close to normal, and the reference group had it somewhat lower $\left(\mathrm{E}_{1-2}=7.72, \mathrm{E}_{2-1}=19.15\right)$.

The value of rigidity of mind before the experiment and after it changed insignificantly in both groups; for the experimental group, it came close to the reference norm $\left(\mathrm{IZ}_{1-1}=0.89\right.$, $\mathrm{IZ}_{2-1}=2.8$, the reference norm being $1.2 ; \mathrm{IZ}_{1-2}=1.45, \mathrm{IZ}_{2-2}=2.72$ ).

Before the experiment, both groups showed a somewhat limited psychological tonus; there could be a feeling of general weakness and fatigue $\left(\mathrm{AS}_{1-1}=2.86, \mathrm{AS}_{2-1}=3.66\right.$, the reference norm being 1.2); after classes, this value was significantly increased in the reference group $\mathrm{AS}_{1-2}=3.19, \mathrm{AS}_{2-2}=5.31$ ). After the experiment, the indicator of restraint of emotions and the mental balance "Myself-Others" for both groups was improved almost twofold; for the experimental group, it was closer to the reference norm $\left(\mathrm{IS}_{1-1}=5.38\right.$, $\mathrm{IS}_{2-1}=6.62, \mathrm{IS}_{1-1}=2.58, \mathrm{IS}_{2-1}=3.34$, the reference norm being 1 ).

\section{Conclusions and recommendations}

As a result of the previous pilot study, it was estimated that using active methods of teaching, unlike the traditional ones, is more efficient in respect of influence on the state of health, adaptability and productivity of mind for students [14].

A series of further experiments with the use of preliminary measurements showed that studying various forms of educational materials causes improvement or deterioration in different groups of values of the current psychological state of students. For example, the value of reserve of control of psychological activity, regulation and adaptation of mind, as well as the value of reserve of syndromological adaptability in the current state of students when self-studying textual material is improved more than that when studying only graphical materials.

The indicators of psychological and physical state of health of students also depend on the form of presentation of the material. For example, the indicators of the emotional sphere of students change during studying various forms of educational materials; there can be an increase in the intensity of emotion, as well as a decrease in vigor in the form of some tendency towards seriousness and apprehension with generally high level of the positive attitude; however, the indicators of mania are decreased and the balance "Myself-Others" is improved. Various forms of educational materials can cause anxiety and increased apprehension, with the textual material contributing the least to this effect.

It is noteworthy that the indicators of a degree of limitation in the current spontaneity and productivity of mind of students when reflecting the reality are improved when studying textual material, whereas the indicators characterizing the risk of presence of actual psycho-traumatizing emotions, as well as a slight limitation of vulnerability and higher sensitivity are deteriorated.

As the experiment result showed, studying textual presentations deteriorates the values of specificity (pragmatism and reality) of thinking, as well as the reality, accuracy and faultless of the opinion.

When studying the illustrated presentations, the indicators of the degree in expressiveness of repeated (obsessive, arising unwillingly) emotions and the decrease in the 
ability to willingly change over to other emotions are improved, whereas the indicators of productivity of mind when reflecting the reality are improved when reading texts.

Basing on our experiment, we can draw the conclusion that working with new educational materials always increases the stress, disregarding of the presentation form of that material. Obviously, in order to achieve the optimal results of the educational process, it is necessary to alternate the presentation forms of the educational material between the textual and the graphical forms; interactive presentations cannot be considered an alternative to studying educational texts.

Despite the fact that educational neurobiology has quite a long history, there is still a lot to be done to implement the results of neuro-studies into the educational process. In order to improve the functioning of the educational system on the basis of the data of neuroscience and better understand their importance for teaching, an open dialogue is required between professionals in these two spheres.One of the directions for cooperation in the inter-disciplinary context "biology- neuro-science - cognitive neuro-science - psychologyeducational studies -organization of educational process" [15].

As BFB technologiesare developed, the focused instrumental monitoring of the state of body and mind and subsequent adaptation to them makes it possible to find out, for example, which time of day and which conditions are the most efficient for teaching a particular person, and tune in the individual regimes of studies, teach the person to selfcontrolling own states (relaxing, concentration etc.) [1].

Further investigations using the methods of biological feedback can help answer the important questions about comprehension of the educational material; about students' motivation, their emotional state and involvement of capabilities to memorize the material; about the optimal time-schedule of classes, alternating between "active" and "passive" hours (for example, between physical training and lectures); about the time required for restoration (relaxation) after difficult classes and exams. Plans for further experiments presume investigations of the role of the teacher in influencing the psychophysiological state of students.

In general, the results of the experiments make it possible to draw the conclusion that using the biological feedback method is efficient from the viewpoint of obtaining the actual data on the current psychological state of students to better tune the parameters of the educational process; on the other side, due to the fact that, during classes, the students run the risk of developing psychological deviations, therefore, to maintain the psychological health and improving the stress-resistance, the method of biological feedback can be used, as well as methods of relieving psycho-emotional stress and BFB training sessions.

The authors of this article would like to acknowledge the assistance from the department "Physical training and adaptation" of the Institute of physical culture, sports and tourism of Peter the Great St. Petersburg Polytechnic University and, personally, Oleg Yevgeniyevich Piskun for the equipment and assistance in carrying out the studies.

\section{References}

1. P. Luksha, D. Peskov, Global Education Futures: Agenda, 206 (2014)

2. M. Yu. Ababkova, V. L. Leontieva, Conflictology: quarterly scientific and practical journal, 1 (2016)

3. Analysis of the state and dynamics of the world market of neurotechnologies [online], Available at: http://sibfrontier.ru/wp-content/uploads/2015/04/RVK_Analizsostoyaniya-i-dinamiki-mirovogo-ryinka-neyrotehnologiy_2015.pdf (2015)

4. O.E. Piskun, The scientific-theoretical journal "Uchenyezapiski", 11 (81) (2011)

5. E.V. Kovalevskaya, Psychological Science and Education, 7 (2015) 
6. T.I. Velichko, Living psychology, 4 (2017)

7. T.S. Vartanova, Application of the biofeedback method in the practice of foreign health care, social assistance and education (SPbJSC "Bio-Svyaz", Saint Petersburg, 2008)

8. P. Ratanasiripong, N. Ratanasiripong, D. Kathalae, ISRN Nursing, 2012 (2012)

9. P. Chaló, A. Pereira, P. Batista, L. Sancho, ApplPsychophysiol Biofeedback, 42 (3) (2017)

10. D. Oman, S. L. Shapiro, C. E. Thoresen, T. G. Plante, T. Flinders, Journal of American college. Health, 56 (2008)

11. V. Strauss, \$1.1 million-plus Gates grants: "Galvanic" bracelets that measure student engagement. The Washington Post [online], Available at: http://www.washingtonpost.com/blogs/answer-sheet/post/11-million-plus-gates-grantsgalvanic-bracelets-that-measure-studentengagement/2012/06/10/gJQAgAUbTV_blog.html (2017)

12. Yu.V. Yumatova, Science and education: a new time, 5 (10) (2015)

13. O.V. Silantieva, Biological feedback technology (BFR) in the work of an educational psychologist. Actual problems of science and education: Theory and practice (Petite, Moscow, 2015)

14. M.Yu. Ababkova, V. L. Leontyeva, Education and science, 7(2017)

15. D.L. Schwartz, K.P. Blair, J.M. Tsang, British Journal of Educational Psychology, II (8) (2012) 